\title{
Red and Orange Colors as Dermoscopic Clues for Fish-Tank Granuloma
}

\author{
Alejandro Lobato-Berezo ${ }^{1,2}$, Gemma Martín-Ezquerra ${ }^{1}$, \\ Ainhoa Vidal-Navarro ${ }^{1}$, Ramón M. Pujol ${ }^{1}$
}

1 Department of Dermatology, Hospital del Mar-Parc de Salut Mar, Barcelona, Spain

2 Department of Dermatology, Hospital Universitari General de Catalunya, Sant Cugat del Vàlles, Barcelona, Spain

Key words: orange, red, nodule, arm, fish-tank granuloma

Citation: Lobato-Berezo A, Martín-Ezquerra G, Vidal-Navarro A, Pujol RM. Red and orange colors as dermoscopic clues for fish-tank granuloma. Dermatol Pract Concept. 2019;9(2):162-164. DOI: https://doi.org/10.5826/dpc.0902a18

Accepted: November 19, 2018; Published: April 30, 2019

Copyright: $@ 2019$ Lobato-Berezo et al. This is an open-access article distributed under the terms of the Creative Commons Attribution License, which permits unrestricted use, distribution, and reproduction in any medium, provided the original author and source are credited.

Funding: None.

Competing interests: The authors have no conflicts of interest to disclose.

Authorship: All authors have contributed significantly to this publication.

Corresponding author: Alejandro Lobato-Berezo, MD, Department of Dermatology, Hospital del Mar, Barcelona, Passeig Marítim, 25-29, 08003 Barcelona, Spain. E-mail: allobe@hotmail.es

\section{Introduction}

We report the case of a 34-year-old white man with an 8-month history of erythematoviolaceous papulonodules distributed from the left hand to the arm in a sporotrichoid pattern.

\section{Case Presentation}

A papulonodule was initially noted on the tip of the patient's fourth left finger. More lesions progressed over the next months along his left arm, with associated tenosynovitis of the same finger. Physical examination revealed several nonconfluent, mildly scaling papulonodules on the dorsal aspect of the left hand, forearm, and arm, with a discrete nonpurulent discharge (Figure 1A). Dermoscopy revealed orange structureless areas associated with white scaling, erosions, serohematic crusts, and dotted/glomerular vessels (Figure 1B). The remaining physical examination was unremarkable.
The patient worked as a waiter and had some fish tanks and a dog with leishmaniasis. A biopsy specimen disclosed mild acanthosis, orthokeratosis, and occasional epidermal erosions (Figure 2A). A diffuse, noncaseating, granulomatous inflammatory infiltrate, composed of lymphocytes, histiocytes, and multinucleated giant cells, was present in the papillary and reticular dermis (Figure 2B). Gram, Ziehl-Neelsen, and PAS stains failed to detect microorganisms. Polymerase chain reactions for both Leishmania spp and Mycobacterium spp were negative. Mycobacterial cultures from a skin biopsy isolated several strains of Mycobacterium marinum.

Our patient started treatment with doxycycline $100 \mathrm{mg}$ once daily for 4 weeks plus clarithromycin $500 \mathrm{mg}$ twice daily for 12 weeks. The lesions disappeared, leaving a mild residual hyperpigmentation.

\section{Discussion}

In dermoscopy, orange color is highly suggestive of dermal granulomas, although it has also been described in non- 


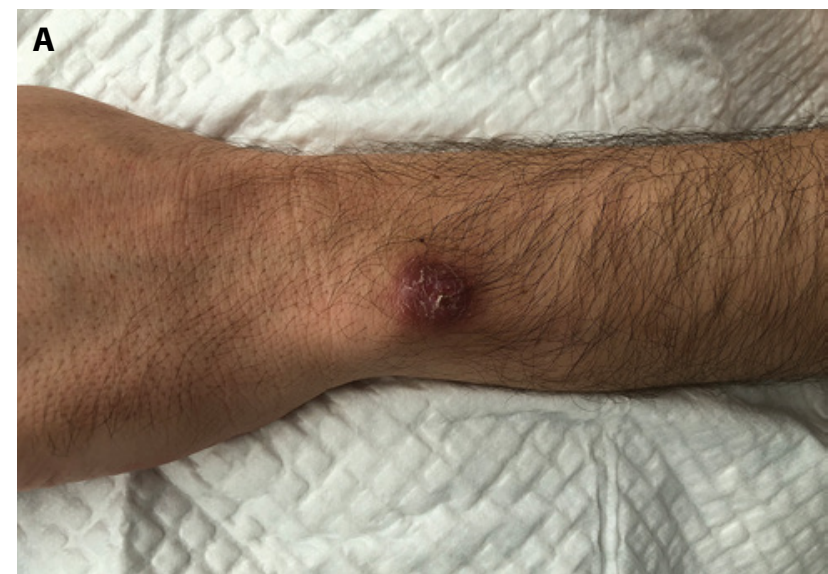

Figure 1. (A) Crusted nodule on the left forearm. (B) Small orange structureless areas associated with white scaling, erosions, serohematic crusts, and dotted/glomerular vessels. [Copyright: (C2019 Lobato-Berezo et al.]

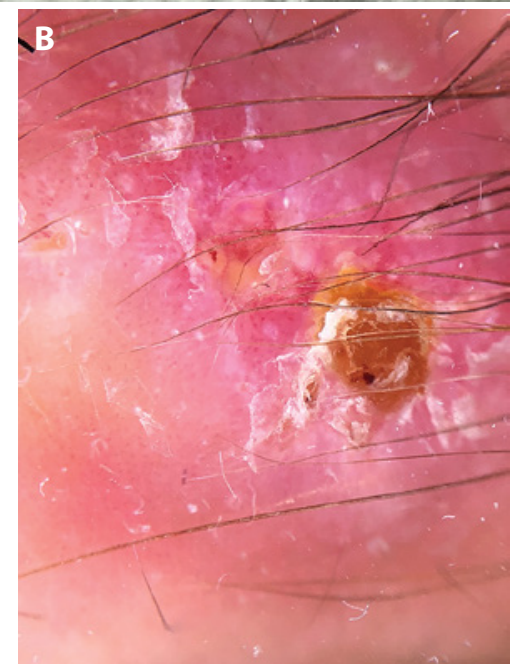

granulomatous diseases [1]. Yellowish to orange color has been the main clue for the description of different patterns in granulomatous diseases. In addition, other dermoscopic features could help in the diagnosis, for example, long and very branched telangiectasias in necrobiosis lipoidica, short telangiectasias and white lines and dots between the translucent orange globules in cutaneous sarcoidosis, unfocused vessels in granuloma annulare, pinkish homogeneous background with no or dull orangish areas in rheumatoid nodules, reddish or purple vascular polygons in granulomatous rosacea, yellow tear drops or white starburst pattern in cutaneous leishmaniasis, follicular openings filled with whitish or yellowish keratotic plugs in acne agminata, white areas with areas of alopecia in borderline tuberculoid leprosy, well-focused linear-branching vessels in lupus vulgaris, or granuloma foreign body reaction. These areas may show focal or diffuse distribution [2]. However, lack of this color does not exclude the diagnosis of cutaneous granulomatous disease, mainly at early stages of the disease or when deep granulomas are present in the reticular dermis.

In this case, white scales could correspond to epidermal orthokeratosis and orange structureless areas to granulomas
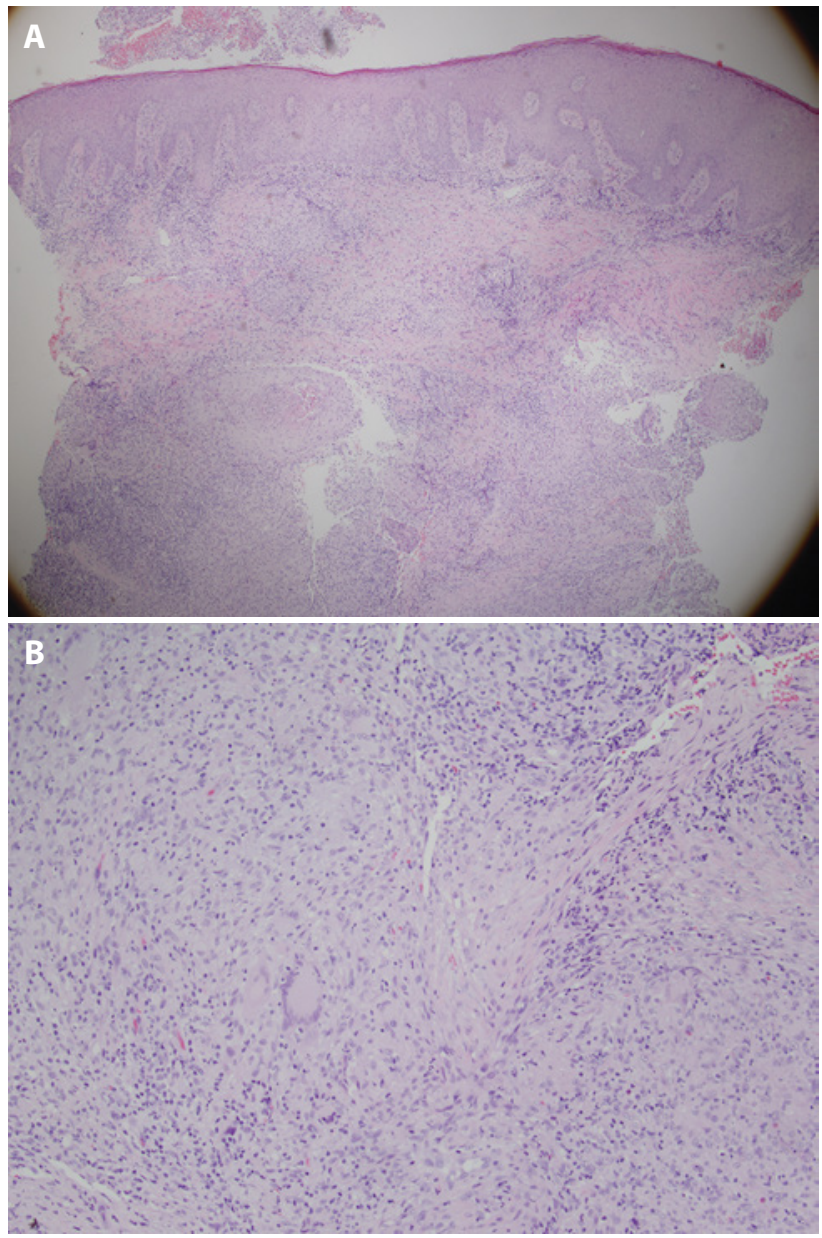

Figure 2. (A) Mild acanthosis, orthokeratosis, and occasional epidermal erosions $(\mathrm{H} \& \mathrm{E}, \times 10)$. (B) Diffuse, noncaseating, granulomatous inflammatory infiltrate, composed of lymphocytes, histiocytes, and multinucleated giant cells $(\mathrm{H} \& \mathrm{E}, \times 40) . \mathrm{H} \& \mathrm{E}=$ hematoxylin and eosin. [Copyright: (92019 Lobato-Berezo et al.]

within the dermis. Dotted and glomerular vessels are secondary to the increased inflammatory granulomatous infiltrate.

On the other hand, histological and even molecular diagnosis of fish-tank granuloma can be challenging. ZiehlNeelsen staining is able to detect acid-fast bacilli in a low number of cases, between $13 \%$ and $50 \%$, depending both on the stage and the presence of tuberculoid granulomas. Culture in Löwenstein-Jensen agar can detect Mycobacterium marinum in $93.3 \%$ of the cases and is still considered one of the most specific diagnostic tools [3-5].

\section{Conclusions}

We present the first description of fish-tank granuloma dermoscopy. We would like to highlight the association of orange structureless areas with dotted/glomerular vessels in the dermoscopy as an additional clue to the clinical lesions and the unilateral location to distinguish it from other granulomatous 
diseases. These dermoscopic findings have been found in a single case and need to be validated in future case series.

\section{References}

1. Bañuls J, Arribas P, Berbegal L, DeLeón FJ, Francés L, Zaballos P. Yellow and orange in cutaneous lesions: clinical and dermoscopic data. J Eur Acad Dermatol Venereol. 2015;29(12):2317-2325.

2. Errichetti E, Stinco G. Dermatoscopy of granulomatous disorders. Dermatol Clin. 2018;36(4):369-375.
3. Belz D, Tantcheva-Poor I, Rasokat H, Fabri M, Schlaak M. Mycobacterium marinum infection initially diagnosed as metastatic Crohn's disease. J Eur Acad Dermatol Venereol. 2016;30(3):514515.

4. Bonamonte D, De Vito D, Vestita M, et al. Aquarium-borne Mycobacterium marinum skin infection: report of 15 cases and review of the literature. Eur J Dermatol. 2013;23(4):510-516.

5. Collina G, Morandi L, Lanzoni A, Reggiani M. Atypical cutaneous mycobacteriosis diagnosed by polymerase chain reaction. $\mathrm{Br} \mathrm{J}$ Dermatol. 2002;147(4):781-784. 